\title{
Efficient Approach for Modeling Collision-Induced Timing Jitter in WDM Return-to-Zero Dispersion-Managed Systems
}

\author{
V. S. Grigoryan, Member, OSA, and A. Richter
}

\begin{abstract}
A semi-analytical approach that allows us to create an efficient numerical algorithm to accurately evaluate collision-induced timing jitter in wavelength-division-multiplexing (WDM) systems with return-to-zero (RZ) modulation format is developed. The approach agrees well with full numerical simulations. The computational time is reduced by two orders of magnitude compared to full numerical simulations.
\end{abstract}

Index Terms-Dispersion management, jitter, optical fiber transmission, wavelength-division multiplexing (WDM).

\section{INTRODUCTION}

I T HAS BEEN widely recognized that massive wavelengthdivision multiplexing (WDM) in dispersion-managed fibers is an efficient method for increasing capacity of fiber communication systems. The best results for the distance bit-rate product in combination with high spectral efficiency have been recently achieved for the return-to-zero (RZ) modulation format in both terrestrial [1] and transoceanic [2], [3] systems. One of the major sources of bit errors in WDM RZ systems is collision-induced timing jitter (CITJ) due to the interchannel cross-phase modulation (XPM). Different approaches have been developed to estimate CITJ [4]-[13]. However, first, they are valid only for the soliton systems and, second, only two-soliton collisions were considered with an exception of [5] where three-soliton collisions were analyzed. Signal pulses in modern WDM RZ systems may significantly differ from both fundamental solitons and dispersion-managed solitons. Besides, as the number of channels in WDM systems is typically larger than three, four and higher number-pulse collisions should be taken into account. Full numerical simulation can be used to fill this gap, however, the computational time increases dramatically with increase of the number of channels $\mathcal{N}$. The computational time grows at least as $\mathcal{N}^{2}$ because one should, first, increase the frequency bandwidth proportionally to $\mathcal{N}$ and, second, reduce the step size in distance proportionally to $\mathcal{N}$ to resolve the fast relative sliding of the channels due to their large group velocity difference. In addition to that, one needs to simulate a long pseudorandom pulse train with a number of bits, marks and spaces, of at least the order of 100 in each channel as a reasonable statistical ensemble

Manuscript received June 8, 1999; revised March 27, 2000. This work was supported by AFOSR.

V. S. Grigoryan is with the Department of Computer Science and Electrical Engineering, University of Maryland Baltimore County, Baltimore, MD 21228 USA.

A. Richter is with Virtual Photonics Incorporated, D 10587 Berlin, Germany. Publisher Item Identifier S 0733-8724(00)06472-0. over which the variance and the average are to be calculated. As a result, full simulation in massive WDM systems becomes so time consuming that it is hardly efficient.

In present paper, we develop a semi-analytical approach for calculating CITJ. The key idea of the approach is to derive an analytical formula for the variance and the average of the pulse central time as a functional of pulse shape evolution in distance in each channel as if each channel is isolated from other channels. We then calculate numerically the evolution of only one signal pulse in each channel by solving nonlinear Schrödinger equation for one pulse, and using the functional formula we calculate numerically the variance and the average of the pulse central time. Finally, we compare the results of our approach with full numerical simulations and find a good agreement. The approach is valid for the systems in which $\mathrm{RZ}$ signal pulses do not spread outside the bit length. In our approach there is no need to process the whole frequency bandwidth of multichannel system, rather we process the bandwidth of only one channel. Besides, in our approach we do not need to simulate propagation of pseudorandom pulse train with large number of marks and spaces, instead we simulate propagation of only one mark. Combination of these two factors allows us to dramatically reduce the computational time by the factor of two orders of magnitude compared to full numerical simulation.

\section{BASIC EQUATIONS}

We start from dynamic equation taking into account the selfphase modulation (SPM) and XPM effects in WDM system

$$
\begin{aligned}
i\left[\frac{\partial}{\partial z}\right. & \left.+\frac{1}{v_{m}(z)} \frac{\partial}{\partial t}\right] q_{m n}+\frac{1}{2} D_{m} \frac{\partial^{2} q_{m n}}{\partial t^{2}} \\
& +\left(\left|q_{m n}\right|^{2}+2 \sum_{k \neq m, l}\left|q_{k l}\right|^{2}\right) q_{m n}=i g(z) q_{m n}
\end{aligned}
$$

where $q_{m n}=E_{m n}\left(n_{2} \omega_{0} L_{D} / A_{\text {eff }} c\right)$ is normalized field amplitude and $E_{m n}$ is the actual field amplitude in the $n$th bit of the $m$ th channel, $n_{2}$ is the Kerr coefficient, $\omega_{0}$ is the central frequency, $L_{D}=t_{0}^{2} / \beta_{0}^{\prime \prime}$ is the dispersion scale length, $t_{0}$ is a scaling time, $\beta_{0}^{\prime \prime}$ is a scaling dispersion, $A_{\text {eff }}$ is the effective fiber cross section, $c$ is the speed of light, $z$ is distance normalized to $L_{D}, t$ is time normalized to $t_{0}, v_{m}(z)=V_{m}(z) t_{0} / L_{D}$ is normalized group velocity and $V_{m}(z)$ is the actual group velocity in the $m$ th channel, $D_{m}(z)$ is the local dispersion $\beta^{\prime \prime}$ normalized with respect to $\beta_{0}^{\prime \prime}, g(z)$ is normalized net gain. We neglected in 
(1) the four-wave mixing effect assuming that the wave vector mismatch is large due to high local dispersion in the fiber. We define the central time $T_{m n}$ and central frequency $\Omega_{m n}$ of the $n$th pulse in the $m$ th channel as

$$
\begin{aligned}
T_{m n} & =\frac{1}{U_{m n}} \int_{-\infty}^{\infty} t_{m}\left|q_{m n}\right|^{2} d t_{m} \\
\Omega_{m n} & =\frac{1}{U_{m n}} \int_{-\infty}^{\infty} \operatorname{Im}\left(\frac{\partial q_{m n}}{\partial t_{m n}} q_{m n}^{*}\right) d t_{m}
\end{aligned}
$$

where $U_{m n}=\int_{-\infty}^{\infty}\left|q_{m n}\right|^{2} d t_{m}$ is the energy of the $n$th pulse in the $m$ th channel, $t_{m}=t-\int_{0}^{z} v_{m}^{-1}\left(z^{\prime}\right) d z^{\prime}$ is the retarded time in the $m$ th channel, and we set $q_{m n}$ to be zero outside the $n$th bit. Differentiating $T_{m n}$ and $\Omega_{m n}$ with respect to $z$ and using (1) we obtain the following dynamic equations for the central time and frequency:

$$
\begin{aligned}
& \frac{d T_{m n}}{d z}=D_{m} \Omega_{m n} \\
& \frac{d \Omega_{m n}}{d z}=\frac{1}{U_{m n}} \int_{-\infty}^{\infty}\left|q_{m n}\right|^{2} \frac{\partial P}{\partial T} d T
\end{aligned}
$$

where

$$
\begin{aligned}
P & =2 \sum_{k \neq m, l}\left|q_{k l}\left(z, T+\theta_{m n, k l}\right)\right|^{2} \\
\theta_{m n, k l} & =\int_{0}^{z}\left[v_{m}^{-1}\left(z^{\prime}\right)-v_{k}^{-1}\left(z^{\prime}\right)\right] d z^{\prime}+T_{m n}-T_{k l}
\end{aligned}
$$

and $T=t_{m n}-T_{m n}$. We neglect the interpulse interference between adjacent pulses in each channel and assume that all signal pulses in each channel have the same shape that may though differ from channel to channel, so that one can write $q_{m n}=\alpha_{m n} q_{m}$, where $\alpha_{m n}$ is the random binary function of $n$ and $m, \alpha_{m n}$ equals 1 for marks and 0 for spaces with the same probability of $1 / 2$, and $q_{m}$ is the shape of signal pulse in the $m$ th channel. Typically, in the dispersion-managed WDM systems there is no significant change of the pulse shape resulting from collisions as the collision distance is much shorter than the nonlinearity scale distance. In this paper we neglect the change of the pulse shape due to the collisions. We will refer to this approximation as elastic collision approximation. However, we take into account evolution of the pulse shape due to the dispersion and SPM effects. Formal solution of (2) and (3) is

$$
\begin{aligned}
T_{m n}(z) & =\int_{0}^{z} D_{m}\left(z^{\prime}\right) \Omega_{m n}\left(z^{\prime}\right) d z^{\prime}+T_{m n}^{0} \\
\Omega_{m n} & =2 \sum_{k \neq m, l} \alpha_{k l} \int_{0}^{z} \frac{S_{m n, k l}\left(z^{\prime}\right)}{U_{m}\left(z^{\prime}\right)} d z^{\prime}
\end{aligned}
$$

where $T_{m n}^{0}$ is the initial central time of the $n$th pulse in the $m$ th channel and the terms

$$
S_{m n, k l}(z)=\int_{-\infty}^{\infty}\left|q_{m}(z, T)\right|^{2} \frac{\partial}{\partial T}\left|q_{k}\left(z, T+\theta_{m n, k l}\right)\right|^{2} d T
$$

describe all possible collisions in the system. Substitution of $\Omega_{m n}$ from (6) into (5) yields

$$
\begin{aligned}
\Delta T_{m n}= & T_{m n}-T_{m n}^{0}=2 \int_{0}^{z} D_{m}\left(z_{1}\right) \\
& \cdot\left[\int_{0}^{z_{1}} \frac{1}{U_{m}\left(z^{\prime}\right)} \sum_{k \neq m, l} \alpha_{k l} S_{m n, k l}\left(z^{\prime}\right) d z^{\prime}\right] d z_{1} .
\end{aligned}
$$

We define the average $\delta T_{m}$ and the variance $\sigma_{m}{ }^{2}$ of the pulse central time in the $m$ th channel as

$$
\begin{aligned}
\delta T_{m} & =\left\langle\Delta T_{m n}\right\rangle=\frac{1}{N} \sum_{n} \Delta T_{m n}, \\
\sigma_{m}^{2} & =\frac{1}{N} \sum_{n}\left(\Delta T_{m n}\right)^{2}-\left(\delta T_{m}\right)^{2},
\end{aligned}
$$

where summation is taken over an ensemble of large number of pulses $N$.

\section{Average Central Time}

Substituting (8) into (9) and taking the average we find that

$$
\delta T_{m}=\int_{0}^{z} D_{m}\left(z_{1}\right)\left[\int_{0}^{z_{1}} \frac{Q_{m}\left(z^{\prime}\right)}{U_{m}\left(z^{\prime}\right)} d z^{\prime}\right] d z_{1}
$$

where

$$
Q_{m}(z)=\frac{2}{N} \sum_{n, k \neq m, l} \alpha_{k l} S_{m n, k l}(z)
$$

We assume that signal pulses are separated enough, so that a pulse in the $m$ th channel interacts only with one pulse in the $k$ th channel which is the nearest to it in the $k$ th channel, while interaction with not nearest pulses in the $k$ th channel is negligible. In fact, we take into account all possible interactions in which only one pulse per channel is taking part. This separation of collisions becomes invalid when RZ pulses expand close to or beyond the limits of the bit slot. However, we note that when RZ pulses expand beyond the bit slots the power of the pulses decreases reducing the cross phase modulation such that CITJ becomes insignificant. Hence, we can still expect that our approach will be valid for the systems with large stretching factors. Substituting (8) into (9) and picking up for each $n$ in the sum over $l$ in (12) only that $l$ th term that corresponds to the nearest bit to the $n$th pulse, we derive

$$
Q_{m}(z)=\sum_{k \neq m} S_{m k}\left(\Theta_{m k}, z\right)
$$

where

$$
\begin{aligned}
S_{m k}(\Theta, z)= & \int_{-\infty}^{\infty}\left|q_{m}(z, T)\right|^{2} \\
& \cdot \frac{\partial}{\partial T}\left|q_{k}\left(z, T+\Theta_{m n}\right)\right|^{2} d T \\
\Theta_{m k}= & \tau_{m k}(z)-J_{m k}(z) T_{0}
\end{aligned}
$$


and

$$
\begin{aligned}
\tau_{m k}(z)= & \int_{0}^{z}\left[v_{m}^{-1}\left(z^{\prime}\right)-v_{k}^{-1}\left(z^{\prime}\right)\right] d z^{\prime} \\
& +\Delta T_{m}^{0}-\Delta T_{k}^{0}+\delta T_{m}-\delta T_{k} \\
J_{m k}(z)= & I N T\left[\tau_{m k}(z) / T_{0}\right]
\end{aligned}
$$

$\Delta T_{i}^{0}$ is the initial time delay in the $i$ th channel relative to the $m$ th channel, so that $T_{i j}^{0}=j T_{0}+\Delta T_{i}^{0}, j=0, \pm 1, \pm 2, \ldots$. Details of the derivation of (13) are described in Appendix A. Fig. 1 shows a schematic diagram of the functions $\tau_{m k}(z)$ and $J_{m k}(z)$ versus distance. Physically, $\tau_{m k}(z)$ means accumulated time delay (or advance) of the $m$ th channel relative to the $k$ th channel, while $J_{m k}(z)$ is the accumulated time delay $\tau_{m k}(z)$ expressed in the number of bit lengths. Using (13) we finally obtain from (11) the following equation for the central time average:

$$
\begin{aligned}
\delta T_{m}= & \sum_{k \neq m} \int_{0}^{z} D_{m}\left(z_{1}\right) \\
& \cdot\left\{\int_{0}^{z_{1}} \frac{S_{m n}\left[\Theta_{m k}\left(z^{\prime}\right), z^{\prime}\right]}{U_{m}\left(z^{\prime}\right)} d z^{\prime}\right\} d z_{1} .
\end{aligned}
$$

\section{Central Time Variance}

From (8) we have

$$
\begin{aligned}
\left\langle\Delta T_{m n}^{2}\right\rangle= & \frac{1}{N} \sum_{n}\left(\Delta T_{m n}\right)^{2} \\
= & \int_{0}^{z} \int_{0}^{z} D_{m}\left(z_{1}\right) D_{m}\left(z_{2}\right) \\
& \cdot\left[\int_{0}^{z_{1}} \int_{0}^{z_{2}} \frac{R_{m}\left(z_{1}^{\prime}, z_{2}^{\prime}\right)}{U_{m}\left(z_{1}^{\prime}\right) U_{m}\left(z_{2}^{\prime}\right)} d z_{1}^{\prime} d z_{2}^{\prime}\right] d z_{1} d z_{2}
\end{aligned}
$$

where

$$
\begin{aligned}
R_{m}\left(z_{1}, z_{2}\right)= & \frac{4}{N} \sum_{n} \sum_{k, k^{\prime} \neq m, l, l^{\prime}} \\
& \cdot \alpha_{k l} \alpha_{k^{\prime} l^{\prime}} S_{m n, k l}\left(z_{1}\right) S_{m n, k^{\prime} l^{\prime}}\left(z_{2}\right) .
\end{aligned}
$$

It is shown in Appendix B that the $R_{m}\left(z_{1}, z_{2}\right)$ term evaluates to

$$
\begin{aligned}
R_{m}\left(z_{1}, z_{2}\right)= & \sum_{k, k^{\prime} \neq m} S_{m k}\left(\Theta_{m k}, z_{1}\right) S_{m k^{\prime}}\left(\Theta_{m k^{\prime}}, z_{2}\right) \\
& +\delta_{J_{m k}^{(1)}, J_{m k}^{(2)}} \sum_{k \neq m} S_{m k}\left(\Theta_{m k}, z_{1}\right) S_{m k}\left(\Theta_{m k}, z_{2}\right)
\end{aligned}
$$

where $J_{m k}^{(1)}=J_{m k}\left(z_{1}\right), J_{m k}^{(2)}=J_{m k}\left(z_{2}\right)$, and $S_{m k}\left(\Theta_{m k}, z\right)$ is defined by (14). Substituting (21) into (19), extracting $\left(\delta T_{m}\right)^{2}$ defined by (11), and integrating in (19) by parts, we obtain

$$
\begin{aligned}
\sigma_{m}^{2}= & \sum_{k \neq m} \int_{0}^{z} \int_{0}^{z} \frac{\mathcal{D}_{m}\left(z_{1}\right) \mathcal{D}_{m}\left(z_{2}\right)}{U_{m}\left(z_{1}\right) U_{m}\left(z_{2}\right)} \\
& \cdot S_{m k}\left(\Theta_{m k}, z_{1}\right) S_{m k}\left(\Theta_{m k}, z_{2}\right) \delta_{J_{m k}^{(1)}, J_{m k}^{(2)}} d z_{1} d z_{2}
\end{aligned}
$$

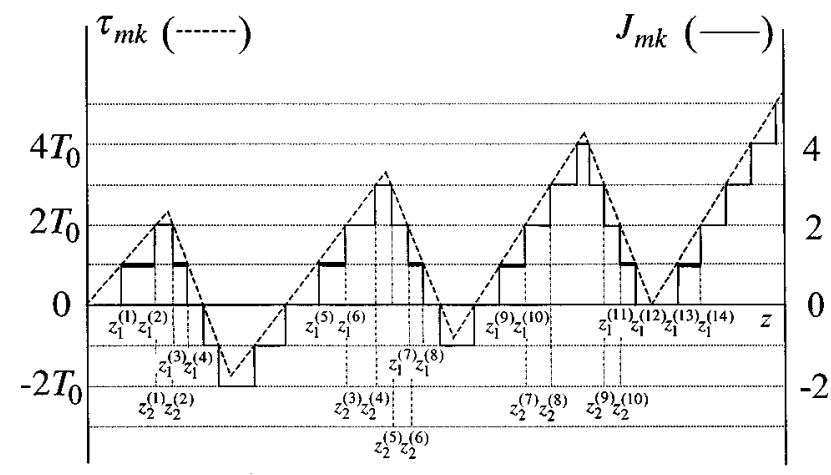

Fig. 1. Schematic plot of the accumulated time delay $\tau_{m k}$, dashed line, and the accumulated time delay $J_{m k}$ expressed in the number of bits, solid line, between the $m$ th and the $k$ th channels versus distance. $\left(z_{J_{m k}}^{(l)}, z_{J_{m k}}^{(l+1)}\right)$, where $l=2 j+1$ and $j=0,1,2, \ldots$ are bit crossing distance intervals in which the accumulated time delay $\tau_{m k}$ is between $J_{m k}$ th and $\left(J_{m k}+1\right)$ th bit, i.e., $J_{m k} T_{0}<\tau_{m k} \leq\left(J_{m k}+1\right) T_{0}$. These intervals cover the whole propagation distance. For a simplicity, only the intervals that correspond to $J_{m k}=1$ and $J_{m k}=2$ are depicted. Bold lines highlight the intervals with $J_{m k}=1$.

where $\mathcal{D}_{m}\left(z_{1,2}\right)=\int_{z_{1,2}}^{z} D_{m}\left(z^{\prime}\right) d z^{\prime}$. Furthermore, each of the integrals in (22) can be represented as

$$
\int_{0}^{z}=\sum_{J_{m k}}\left(\sum_{l} \int_{z_{J_{m k}}^{(l)}}^{z_{J_{m k}}^{(l+1)}}\right)
$$

where $\left(z_{J_{m k}}^{(l)}, z_{J_{m k}}^{(l+1)}\right)$ with $l=2 j+1$ and $j=0,1,2, \ldots$ are bit crossing distance intervals in which the accumulated time delay between the $m$ th and the $k$ th channels $\tau_{m k}$ is between $J_{m k}$ th and $\left(J_{m k}+1\right)$ th bit, i.e., $J_{m k} T_{0}<\tau_{m k} \leq\left(J_{m k}+1\right) T_{0}$, see Fig. 1. We note that these intervals cover the whole transmission distance. Substituting (23) into (22) and keeping only the terms with $J_{m k}^{(1)}=J_{m k}^{(2)}$ we finally obtain the following formula for the central time variance:

$$
\sigma_{m}^{2}=\sum_{k \neq m, J_{m k}}\left[\sum_{l} \int_{z_{J_{m k}}^{(l)}}^{z_{J_{m k}}^{(l+1)}} \frac{\mathcal{D}_{m}\left(z_{1}\right)}{U_{m}\left(z_{1}\right)} S_{m k}\left(\Theta_{m k}, z_{1}\right) d z_{1}\right]^{2}
$$

where summation with respect to $l$ is taken over $l=2 j+1$ with $j=0,1,2, \ldots$, while summation with respect to $J_{m k}$ is taken over $J_{m k}=0, \pm 1, \pm 2, \pm 3, \ldots$

\section{Discussion AND COMPARISON WITH FULl SimUlations}

Equations (18) and (24) constitute a complete set of equations for determining the average and the variance of the pulse central time. This is the key result of the paper. Equations (18) and (24) are functionals of a signal pulse shape in each channel. Within the elastic collision approximation discussed in Section I, evolution of the pulse shape $\left|q_{k}(z, T)\right|^{2}$ in distance $z$ and time $T$ in each $m$ th channel is determined by the nonlinear Schrödinger equation

$$
i \frac{\partial q_{k}}{\partial z}+\frac{1}{2} D_{k} \frac{\partial^{2} q_{k}}{\partial T^{2}}+\left|q_{k}\right|^{2} q_{k}=i g(z) q_{k} .
$$

In order to calculate CITJ in our approach, we first follow the evolution of the pulse shape $\left|q_{k}(z, T)\right|^{2}$ in each $k$ th channel by 


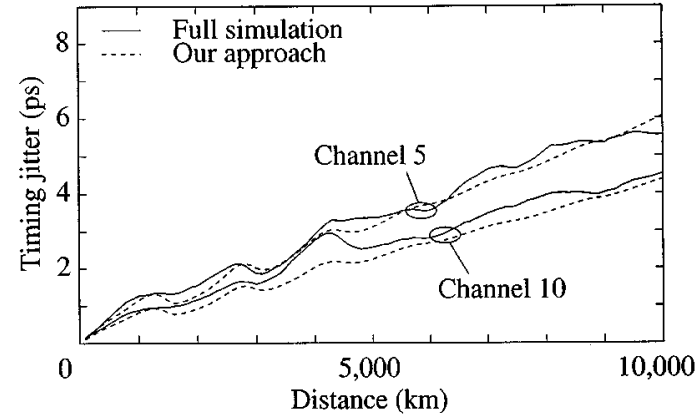

Fig. 2. Dependence of collision-induced timing jitter, $\sigma_{m}$, on distance for the middle, $m=5$, and the outer, $m=10$, channels in 10 channel system. Solid line is the result of full simulations, dashed line is the result of our approach. The dispersion map parameters are: $d_{1}=4.0 \mathrm{ps} / \mathrm{nm}-\mathrm{km}, L_{1}=95.6 \mathrm{~km}$, $d_{2}=-85 \mathrm{ps} / \mathrm{nm}-\mathrm{km}$, and $L_{2}=4.4 \mathrm{~km}$.

numerically solving the nonlinear Schrödinger equation (25) for only one signal pulse (mark). Then, by numerical integration in (18), we calculate the average of the pulse central time $\delta T_{m}$ in each channel. Finally, using (24) and a simple numerical algorithm for calculating the sum of intergals over the bit crossing distance intervals $\left(z_{J_{m k}}^{(l)}, z_{J_{m k}}^{(l+1)}\right)$ we evaluate the variance of the pulse central time $\sigma_{m}^{2}$. To validate our approach we compare it with full numerical simulations of 10 channel WDM system with $10 \mathrm{~Gb} / \mathrm{s}$ bit rate in each channel with different dispersion maps, channel spacings, and amplifier arrangements by launching 128 bit period pseudorandom pulse train in each channel from the midpoint of the anomalous dispersion span. The dispersion map period consists of a span of the anomalous dispersion fiber with the dispersion $d_{1}$ with the length $L_{1}$ and a span of the dispersion compensated fiber with the dispersion $d_{2}$ and the length $L_{2}$. The pulse duration of the initial signal pulses is taken $16.74 \mathrm{ps}$ unless otherwise specified. CITJ turns to be different for different channels even when each channel has the same dispersion. To evaluate this difference we, first, consider the systems with zero dispersion slope.

Fig. 2 shows result of the comparison for a system with amplifiers located at the midpoints of the anomalous and normal dispersion spans, $50 \mathrm{~km}$ appart. The channel spacing is 100 $\mathrm{GHz}$, initial time shift of adjacent channels is $50 \mathrm{ps}$. The peak power of the initial pulses is $3.13 \mathrm{~mW}$. In Fig. 2, the middle 5th channel experiences stronger timing jitter compared to the outer 10th channel because the middle channel sees more collisions from the slowly moving neighboring channels. The middle channel has twice more nearest neighboring channels than the outer channel has. Channel 1 has the same timing jitter that of channel 10, the values of CITJ for all other intermidiate channels lie in between the timing jitter of the fifth and the tenth channels. There is a good agreement between our approach and full numerical simulations. Fig. 2 shows that the growth of CITJ in distance is not a monotonous function, it has periodically spaced humps. The humps are quite clearly visible up to $6000 \mathrm{~km}$ however, after $6000 \mathrm{~km}$ they degrade. Our analysis shows that the period of the humps $L_{h}$ is equal to the bit passing distance for adjacent channels with respect to the average dispersion, i.e. $L_{h}=T_{b} /\left(\overline{\beta^{\prime \prime}} \Delta \omega\right)$, where $T_{b}$ is the bit length, $\Delta \omega$ is the channel separation, and $\overline{\beta^{\prime \prime}}$ is the average dispersion expressed in the ac-
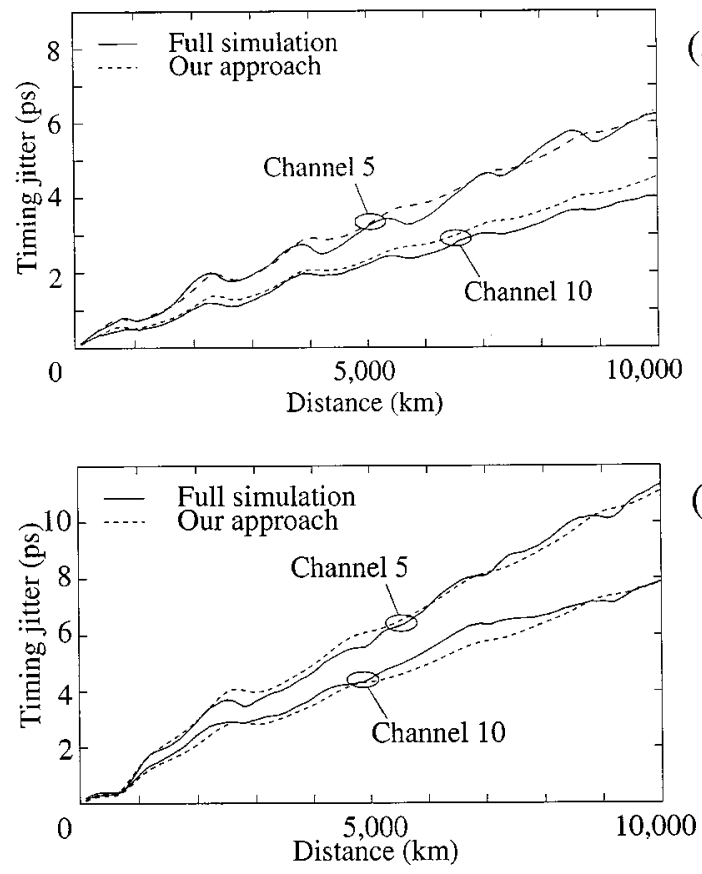

Fig. 3. Dependence of collision-induced timing jitter, $\sigma_{m}$, on distance for the middle, $m=5$, and the outer, $m=10$, channels in ten-channel system. Solid line is the result of full simulations, dashed line is the result of our approach. The dispersion map parameters are: $d_{1}=3.0 \mathrm{ps} / \mathrm{nm}-\mathrm{km}, L_{1}=93 \mathrm{~km}, d_{2}=$ $-38.741 \mathrm{ps} / \mathrm{nm}-\mathrm{km}$, and $L_{2}=7 \mathrm{~km}$. The channel spacing is (a) $100 \mathrm{GHz}$, (b) $75 \mathrm{GHz}$.

tual units. In the case of Fig. $2 L_{h}=1600 \mathrm{~km}$. It follows from this formula that the period of humps will scale inversily proportional to the channel spacing for different dispersion map with the same average dispersion.

This formula is confirmed by two other examples of 10 channel $\times 10 \mathrm{~Gb} / \mathrm{s}$ system illustrated in Figs. 3 and 4. The dispersion map in Fig. 3(a) and Fig. 3(b) is the same. Amplifiers are located at the midpoints of normal and anomalous spans 50 $\mathrm{km}$ apart. The peak power of the initial pulses in Fig. 3(a) and Fig. 3(b) is the same, $2.9 \mathrm{~mW}$. Initial time shifts of adjacent channels are $50 \mathrm{ps}$ for both cases in Fig. 3. The channel spacing in Fig. 3(a) and Fig. 3(b) is 100 and $75 \mathrm{GHz}$, respectively. The accumulated timing jitter in Fig. 3(b) is about twice as large as the timing jitter in Fig. 3(a) due to smaller channel spacing. In the case of Fig. 3(a) the period of the humps is about $L_{h}=1600$ km, while in the case of Fig. 3(b) it is about $L_{h}=2133 \mathrm{~km}$ that is in coplience with inverse proportionality of the period on the channel spacing according to abovementioned formula. The period of humps does not depend on dispersion map at fixed average dispersion and channel spacing. This is illustrated by Fig. 4 where the average dispersion and channel spacing are the same as in Fig. 3(b) but the dispersion map is different. In Fig. 4 amplifiers are located $50 \mathrm{~km}$ apart at the midpoints and at the edges of normal and anomalous spans. The peak power of the initial pulses in Fig. 4 is $2.8 \mathrm{~mW}$, initial time shifts of adjacent channels are 50 ps. The period of the humps is $L_{h}=2133 \mathrm{~km}$, which is the same as in Fig. 3(b). Note that the accumulated timing jitter in Fig. 4 is also about the same as the timing jitter in Fig. 3(b). We note that in all three cases shown 


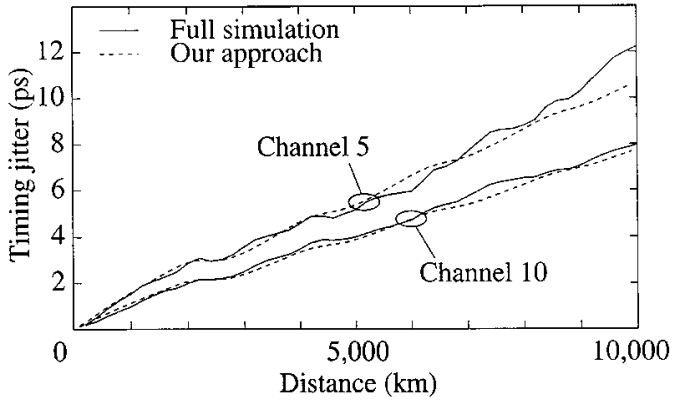

Fig. 4. Dependence of collision-induced timing jitter, $\sigma_{m}$, on distance for the middle, $m=5$, and the outer, $m=10$, channels in ten-channel system. Solid line is the result of full simulations, dashed line is the result of our approach. The dispersion map parameters are: $d_{1}=2.3445 \mathrm{ps} / \mathrm{nm}-\mathrm{km}, L_{1}=100 \mathrm{~km}$, $d_{2}=-2.1882 \mathrm{ps} / \mathrm{nm}-\mathrm{km}$, and $L_{2}=100 \mathrm{~km}$. The channel spacing is $75 \mathrm{GHz}$.

in Figs. 3 and Fig. 4 the period of the humps agrees well with full simulations up to $5000 \mathrm{~km}$. Although for larger distances there is some discrepancy between the curves the agreement remains reasonably good.

CITJ may significantly depend on the initial interchannel time delay. This problem has been well recognized in [14] and [4] for the soliton systems. As far as different sources are typically used for different channels, the pulse trains in different channels are not mutually synchronized allowing a slow relative drift in time in the interchannel time delays. This drift results in a variation of the collision-induced timing jitter. Evaluation of this variation of CITJ based on full numerical simulation is even much more time consuming than the full simulation of CITJ at fixed interchannel time delays, because one should repeat the calculation of CITJ for a large number of different realizations of the interchannel time delays. Using our approach we explore this effect assuming that the initial interchannel time delays are random parameters that are statistically independent and uniformly distributed within the bit period of 100 ps. Fig. 5 shows the average (center solid line) and the variation of CITJ (shadow space around the solid line between two dashed lines) for 5 th channel over 100 realizations of the initial interchannel time delays. The upper dashed line represents the average of $\sigma_{5}$ plus the standard deviation of $\sigma_{5}$ while the lower dashed line represents the average of $\sigma_{5}$ minus the standard deviation of $\sigma_{5}$. The standard deviation is calculated over 100 relaizations of the initial interchannel time delays. The system parameters in Fig. 5 are the same that in Fig. 2 but the amplifiers are placed at only the midpoints of the anomalous dispersion spans with the amplifier distance of 100 $\mathrm{km}$. The peak power of the initial pulses is $5.78 \mathrm{~mW}$. Fig. 5 shows that at $100 \mathrm{GHz}$ channel spacing the spread in CITJ at $10000 \mathrm{~km}$ is about 3 ps while the average timing jitter is $7.6 \mathrm{ps}$. However, this spread doubles and becomes significant when the channel spacing reduces to $75 \mathrm{GHz}$. Fig. 6 shows a good agreement of our approach with full simulations of CITJ for the same system parameters that in Fig. 5(a) and the interchannel time delays for which CITJ was minimum over 100 realizations of the initial interchannel time delays processed in Fig. 5(a).

In all the cases considered above RZ pulses did not expand beyond their bit slots. In Fig. 7 we show results for a Tyco type [2] system in which RZ pulses expand over several bit slots.
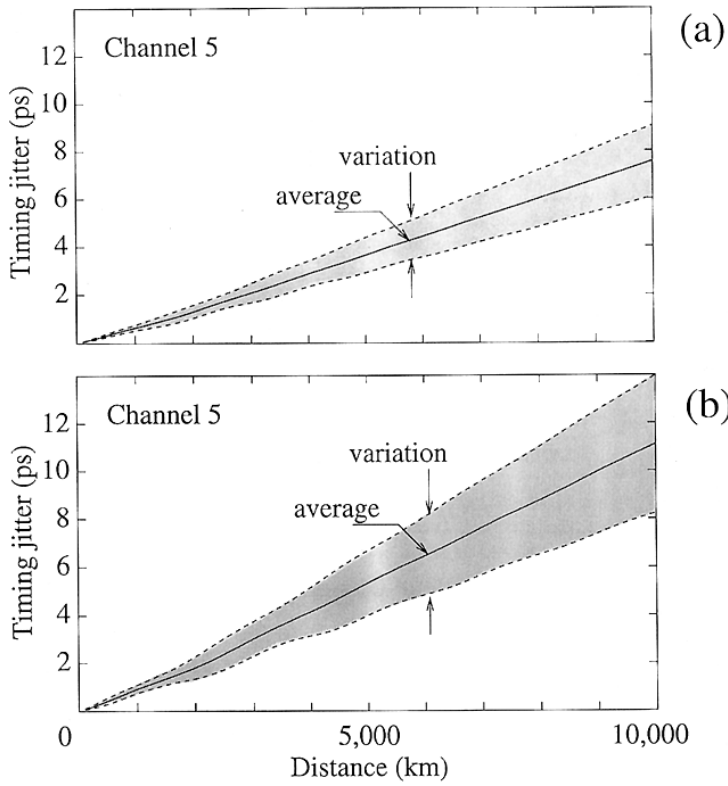

(b)

Fig. 5. The average (center solid line) and the variation of CITJ, $\sigma_{5}$, (shadow space around the solid line between two dashed lines) for fifth channel over 100 realizations of the initial interchannel time delays. The variation designates a spread in the central time variance, so that the upper dashed line represents the average of $\sigma_{5}$ plus the standard deviation of $\sigma_{5}$ while the lower dashed line represents the average of $\sigma_{5}$ minus the standard deviation of $\sigma_{5}$. The standard deviation of $\sigma_{5}$ is calculated over 100 relaizations of the initial interchannel time delays. The system parameters in Fig. 4 are the same that in Fig. 2 but the amplifiers are placed at only the midpoints of the anomalous dispersion spans. The amplifier distance is $100 \mathrm{~km}$, the channel spacing is (a) $100 \mathrm{GHz}$ and (b) $75 \mathrm{GHz}$

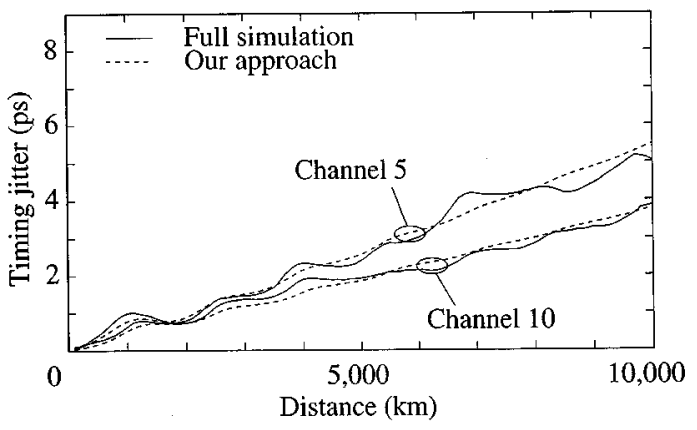

Fig. 6. Plots of CITJ, $\sigma_{m}$, for the 5th, $m=5$, and the tenth, $m=10$, channels versus distance. All system parameters are the same that in Fig. 4(a). The interchannel time delays relative to the fifth channel from channel 1 through channel 10 are: $-26,46.7,10,-4.2 \mathrm{ps},-33.2 \mathrm{ps}, 25 \mathrm{ps}, 20 \mathrm{ps},-9.2,42.7,20.7$ $\mathrm{ps}$, respectively. This realization of the interchannel time delays is one of 100 realizations processed in Fig. 4(a) for which CITJ was minimum.

The pulse duration of the initial signal pulses is $50 \mathrm{ps}$, and the peak power is $0.6 \mathrm{~mW}$. The channel spacing is $100 \mathrm{GHz}$, the dispersion map length is $495 \mathrm{~km}$, and the amplifier spacing is 45 km. Remarkably that Fig. 7 shows a good agreement between our approach and full simulations. The results agree with our assumption made in Section III that when RZ pulses expand significantly then the power of the pulses decreases, reducing the cross phase modulation such that contribution of collisions of these pulses to CITJ is small and, consequently, our approach remains valid. 


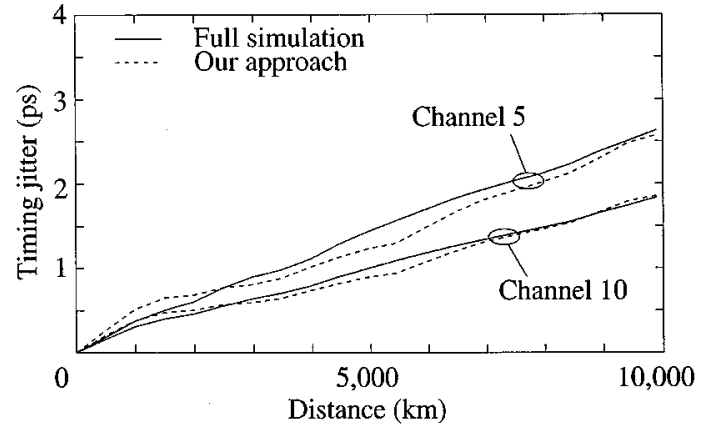

Fig. 7. Dependence of collision-induced timing jitter, $\sigma_{m}$, on distance for the middle, $m=5$, and the outer, $m=10$, channels in 10 channel system applying chirped return-to-zero modulation format. Solid line is the result of full simulations, dashed line is the result of our approach. The dispersion map parameters are: $d_{1}=-2.0 \mathrm{ps} / \mathrm{nm}-\mathrm{km}, L_{1}=450 \mathrm{~km}, d_{2}=19.78 \mathrm{ps} / \mathrm{nm}-\mathrm{km}$, and $L_{2}=45 \mathrm{~km}$.

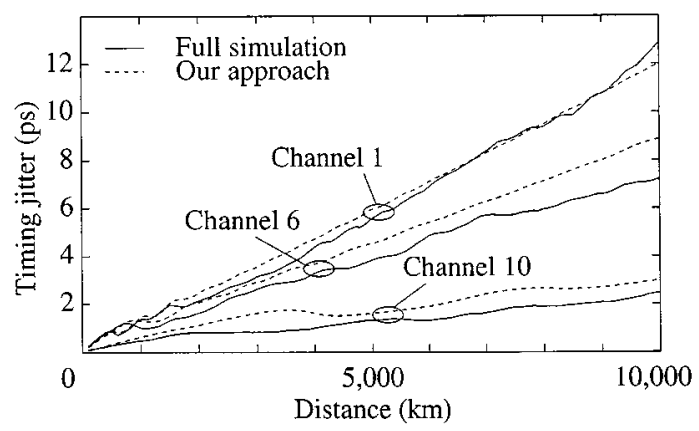

Fig. 8. Dependence of CITJ, $\sigma_{m}$, on distance for a system with nonzero dispersion slope. The arrangement of amplifiers, channel spacing and initial interchannel time delays are the same that in Fig. $2 . d_{1}=4.0 \mathrm{ps} / \mathrm{nm}-\mathrm{km}$, $L_{1}=95.66 \mathrm{~km}, d_{2}=-85 \mathrm{ps} / \mathrm{nm}-\mathrm{km}$, and $L_{2}=4.34 \mathrm{~km}$. The dispersion slope in the anomalous span is $0.04 \mathrm{ps} / \mathrm{nm}^{2}-\mathrm{km}$ while the dispersion slope in the normal span is $-0.2 \mathrm{ps} / \mathrm{nm}^{2}-\mathrm{km}$.

Finally, we show in Fig. 8 dependence of CITJ on distance for a system with nonzero dispersion slope. In channel 1 the average dispersion is maximum, $0.244 \mathrm{ps} / \mathrm{nm}-\mathrm{km}$, while in channel 10 the average dispersion is minimum, $0.03 \mathrm{ps} / \mathrm{nm}-\mathrm{km}$. As a result the timing jitter in channel 1 is significantly larger than in channel 10. We launched pulses with different peak powers in different channels to compensate for the nonlinearity, the peak power of initial pulses from channel 1 through channel 10 are $8,7.2,6.4,5.6,4.8,4,3.3,2.5,1.72$, and $1 \mathrm{~mW}$, respectively. In Fig. 8 there is a good agreement of our approach with full simulations for channels 1 and 10 and a reasonable agreement for channel 6. We note that the humps are not visible in Fig. 8 any more as the bit passing distance periodicity is violated as different channels have different average dispersions.

\section{CONCLUSION}

In conclusion, we developed a semi-analytical approach that allows us to create an efficient algorithm to accurately estimate collision-induced timing jitter in RZ WDM in which RZ signal pulses do not spread outside the bit length. There is a good agreement of our approach with full numerical simulations. Functional dependence of collision-induced timing jitter on the shape of signal pulses derived analytically reduces the computational time by two orders of magnitude compared to full numerical simulations. We use our approach to evaluate the spread in the variance of the pulse central time due to uncontrolled interchannel time delays at the input of a system. We show that this spread may result in a significant performance impairment of a dispersion-managed system.

\section{APPENDIX A}

Fixing $k$ in the sum (12) we pick up for each $n$ only one $l$ term with $l=n+J_{m k}$ that corresponds to the nearest bit in the $k$ th channel to the $n$th bit in the $m$ th channel. Then $Q_{m}(z)$ becomes

$$
\begin{aligned}
Q_{m}(z)= & \frac{2}{N} \sum_{k \neq m, n} \alpha_{k, n+J_{m k}} S_{m, n ; k, n+J_{m k}} \\
& \cdot\left(\Theta_{m, k}+X_{m, n}-X_{k, n+J_{m k}}, z\right) \\
= & \sum_{k \neq m}\left\langle S_{m, n ; k, i+J_{m k}}\left(\Theta_{m, k}+X_{m, i}-X_{k, i+J_{m k}}, z\right)\right\rangle
\end{aligned}
$$

where the angle braces mean the average over all $i$ for which $\alpha_{k, i+J_{m k}}=1, X_{i j}=\Delta T_{i j}-\delta T_{i}$ are the deviations from the pulse central time, and we took into account that $\sum_{i} \alpha_{k, i} / N=$ $1 / 2$. Expanding (A1) into the series of $\Delta X=X_{m, i}-X_{k, i+J_{m k}}$ and averaging we obtain that up to the small corrections of the order of $O\left(\langle\Delta X\rangle^{4}\right)$

$$
\begin{aligned}
Q_{m}(z)= & \sum_{k \neq m}\left[S_{m k}\left(\Theta_{m k}, z\right)\right. \\
& \left.+\frac{1}{2} \frac{\partial^{2} S_{m k}\left(\Theta_{m k}, z\right)}{\partial \Theta_{m k}^{2}}\left(\sigma_{m}^{2}-\sigma_{k}^{2}\right)\right]
\end{aligned}
$$

where

$$
\begin{aligned}
S_{m k}\left(\Theta_{m k}, z\right) & =\int_{-\infty}^{\infty}\left|q_{m}(z, T)\right|^{2} \frac{\partial}{\partial T}\left|q_{k}\left(z, T+\Theta_{m k}\right)\right|^{2} d T \\
\Theta_{m k} & =\tau_{m k}(z)-J_{m k}(z) T_{0}
\end{aligned}
$$

$\sigma_{n}^{2}=\left\langle X_{n, i}^{2}\right\rangle$ is the variance of the pulse central time in the $n$th channel. In deriving (A2) we took into account that from the symmetry considerations $\langle\Delta X\rangle=\left\langle(\Delta X)^{3}\right\rangle=0$. Though a complete formula (A2) can be used to derive the central time average, we note that the second term in the right hand side of (A2) has typically a small contribution. Indeed, for the $k$ th channels adjacent to the $m$ th channel $\sigma_{m}^{2}$ is close to $\sigma_{k}^{2}$, so that $\sigma_{m}^{2}-\sigma_{k}^{2}$ is small, for the far spaced $k$ th channels $\sigma_{m}^{2}-\sigma_{k}^{2}$ is not small, however, CITJ induced by the far spaced channels is assumed to be small. In the Section III we neglect the second term in the right-hand side of (A2).

\section{APPENDIX B}

Fixing $k$ and $k^{\prime}$ in (20) we pick up for each $n$ only one $l$ term with $l=n+J_{m k}$ and only one $l^{\prime}$ term with $l^{\prime}=n+J_{m k}$

$$
\begin{aligned}
R_{m}\left(z_{1}, z_{2}\right)= & \frac{4}{N} \sum_{k, k^{\prime} \neq m, n} \alpha_{k, n+J_{m k}^{(1)}} \alpha_{k^{\prime}, n+J_{m k^{\prime}}^{(2)}} \\
& \times S_{m, n ; k, n+J_{m k}^{(1)}}\left[\Theta_{m k}\left(z_{1}\right)+\Delta X_{1}, z_{1}\right] \\
& \cdot S_{m, n ; k^{\prime}, n+J_{m k^{\prime}}^{(2)}}\left[\Theta_{m k^{\prime}}\left(z_{2}\right)+\Delta X_{2}, z_{2}\right]
\end{aligned}
$$


where

$$
\begin{aligned}
& \Delta X_{1}=X_{m, n}\left(z_{1}\right)-X_{k, n+J_{m k}^{(1)}}\left(z_{1}\right) \\
& \Delta X_{2}=X_{m, n}\left(z_{2}\right)-X_{k^{\prime}, n+J_{m k}^{(2)}}\left(z_{2}\right)
\end{aligned}
$$

and

$$
X_{i j}(z)=\Delta T_{i j}(z)-\delta T_{i}(z)
$$

Furthermore, we note that as the marks and spaces in different channels are mutually independent one can write

$$
\frac{4}{N} \sum_{n} \alpha_{k, n+J_{m k}^{(1)}} \alpha_{k^{\prime}, n+J_{m k^{\prime}}^{(2)}}=1+\delta_{k k^{\prime}} \delta_{J_{m k}^{(1)}, J_{m k^{\prime}}^{(2)}} \cdot
$$

Expanding (B1) into the series of $\Delta X_{1}$ and $\Delta X_{2}$, neglecting the second order terms and taking into account (B2) we obtain

$$
\begin{aligned}
R_{m}\left(z_{1}, z_{2}\right)= & \sum_{k, k^{\prime} \neq m} S_{m k}\left[\Theta_{m k}\left(z_{1}\right), z_{1}\right] \\
& \cdot S_{m k^{\prime}}\left[\Theta_{m k^{\prime}}\left(z_{2}\right), z_{2}\right] \\
& +\delta_{J_{m k}^{(1)}, J_{m k^{\prime}}^{(2)}} \sum_{k \neq m} S_{m k}\left[\Theta_{m k}\left(z_{1}\right), z_{1}\right] \\
& \cdot S_{m k^{\prime}}\left[\Theta_{m k^{\prime}}\left(z_{2}\right), z_{2}\right]
\end{aligned}
$$

where $J_{m k}^{(1)}=J_{m k}\left(z_{1}\right), J_{m k}^{(2)}=J_{m k}\left(z_{2}\right)$, and $S_{m k}\left[\Theta_{m k}(z), z\right]$ is defined by (14).

\section{ACKNOWLEDGMENT}

The authors would like to acknowledge all numerical calculations in this work have been performed using "Photonic Transmission Design Suite" (PTDS) software tool of Virtual Photonics, Inc. They are also grateful to C. R. Menyuk for his support and fruitful discussions and to M. Horowitz for his useful comments.

\section{REFERENCES}

[1] D. Le Guen, S. Del Burgo, M. L. Moulinard, D. Grot, M. Henry, F. Favre, and T. Georges, "Narrow band $1.02 \mathrm{Tbit} / \mathrm{s}(51 \times 20 \mathrm{Gbit} / \mathrm{s})$ soliton DWDM transmission over $1000 \mathrm{~km}$ of standard fiber with $100 \mathrm{~km}$ amplifier spans," in Proc. Optical Fiber Communication Conf., 1999 OSA Tech. Dig. Ser, Postdeadline Papers. Washington, D.C., 1999, Postdeadline Paper PD4.
[2] N. S. Bergano, C. R. Davidson, C. J. Chen, B. Pederson, M. A. Mills, N. Ramanujam, H. D. Kidorf, A. B. Puc, M. D. Levonas, and H. Abdelkader, " $640 \mathrm{~Gb} / \mathrm{s}$ transmission of sixty-four $10 \mathrm{~Gb} / \mathrm{s}$ WDM channels over $7200 \mathrm{~km}$ with 0.33 (bits/s)/Hz spectral efficiency," in Proc. Optical Fiber Communication Conf., 1999 OSA Tech. Dig. Ser., Postdeadline Papers. Washington, D.C., 1999, Postdeadline Paper PD2.

[3] K. Imai, T. Tsuritani, N. Takeda, K. tanaka, N. Edagawa, and M. Suzuki, "500 Gb/s $(50 \times 10 \mathrm{~Gb} / \mathrm{s})$ WDM transmission over $4000 \mathrm{~km}$ using broadband EDFA's and low dispersion slope fiber," in Proc. Optical Fiber Communication Conf., 1999 OSA Tech. Dig. Ser., Postdeadline Papers. Washington, DC, 1999, Postdeadline Paper PD5.

[4] L. F. Mollenauer, S. G. Evangelides, and J. P. Gordon, "Wavelength division multiplexing with solitons in ultra-long distance transmission using lumped amplifiers," J. Lightwave Technol., vol. 9, pp. 362-367, 1991.

[5] S. G. Evangelides, Jr. and J. P. Gordon, "Energy transfer and frequency shifts from three soliton collisions in a multiplexed transmission line with periodic amplification," J. Lightwave Technol., vol. 14, pp. 1639-1645, 1996.

[6] H. Sugahara, H. Kato, and Y. Kodama, "Maximum reductions of collision induced frequency shift in soliton-WDM systems with dispersion compensation," Electron. Lett., vol. 33, pp. 1065-1066, 1997.

[7] J. F. L. Devaney, W. Forysiak, A. M. Niculae, and N. J. Doran, "Soliton collisions in dispersion-managed wavelength-division-multiplexed systems," Opt. Lett., vol. 22, pp. 1695-1697, 1997.

[8] A. Mecozzi, "Timing jitter in wavelength-division-multiplexed filtered soliton transmission," J. Opt. Soc. Amer. B, vol. 15, pp. 152-161, 1998.

[9] T. Hirooka and A. Hasegawa, "Chirped soliton interaction in strongly dispersion-managed wavelength-division-multiplexed system," Opt. Lett., vol. 23, pp. 768-770, 1998.

[10] A. M. Niculae, W. Forysiak, A. J. Gloag, J. H. B. Nijhof, and N. J. Doran, "Soliton collisions with wavelength-division multiplexed systems with strong dispersion management," Opt. Lett., vol. 23, pp. 1354-1356, 1998.

[11] H. Sagahara, A. Maruta, and Y. Kodama, "Optimal allocation of amplifiers in a dispersion-managed line for a wavelength-division-multiplexed soliton transmission system," Opt. Lett., vol. 24, pp. 145-147, 1999.

[12] H. Sagahara, H. Kato, T. Inoue, A. Maruta, and Y. Kodama, "Optimal dispersion management for a wavelength-division-multiplexed optical soliton transmission system," J. Lightwave Technol., vol. 17, pp. 1547-1559, 1999.

[13] M. J. Ablowitz, G. Biondini, S. Chakvaraty, and R. L. Horne, "On timing jitter in wavelength-division-multiplexed soliton systems," Opt. Commun., vol. 150, pp. 305-318, 1998.

[14] B. A. Andrekson et al., "Soliton collision interaction force dependence on wavelength separation in fiber amplifier based systems," Electron. Lett., vol. 26, pp. 1499-1501, 1990.

V. S. Grigoryan, photograph and biography not available at the time of publication.

A. Richter, photograph and biography not available at the time of publication. 